Conclusions In healthy volunteers, Talnetant had no significant effect on cough reflex sensitivity to citric acid despite an adequately powered study and sufficient systemic drug exposure. Possible explanations for this lack of efficacy are (1) NK3 receptors do not play an appreciable role in the healthy human cough reflex or (2) the role of NK3 receptors may be limited to the central nervous system where Talnetant has limited penetrance and receptor occupancy. Whilst this study suggests predominantly peripherally acting NK3 receptor antagonists do not influence the cough reflex in healthy volunteers, a significant effect in patients with a hypersensitive cough reflex cannot be excluded and this class of drugs may yet prove to have anti-tussive properties, especially with improved central activity.

\section{S115 MAGNITUDE OF COUGH RESPONSE TO INHALED CAPSAICIN DIFFERENTIATES BETWEEN HEALTH AND DISEASE}

doi:10.1136/thx.2010.150946.16

${ }^{1} \mathrm{E} C$ Young, ${ }^{1} \mathrm{H}$ Sumner, ${ }^{2} \mathrm{~A}$ Kelsall, ${ }^{1} \mathrm{~A}$ A Woodcock, ${ }^{1} \mathrm{~J}$ A Smith. ${ }^{1}$ University of Manchester, Manchester, UK; ${ }^{2}$ University Hospitals South Manchester NHS Foundation Trust, Manchester, UK

Background Patients with chronic cough (CC) have substantially higher 24-h cough rates compared to patients with asthma (A) or healthy controls (HC). CC patients also have a more sensitive cough reflex, when measured as the 'threshold' dose of a tussive agent (eg, capsaicin) inducing at least two or five coughs (C2/C5), although there is substantial overlap between CC and HC. We aimed to investigate if the 'magnitude' of cough response to inhaled capsaicin would better differentiate CC patients from A and HC subjects.

Methods We devised a cough challenge technique, with increasing doubling concentrations of capsaicin $(0.48-1000 \mu \mathrm{Mol})$ extended beyond the C5 threshold, up to the maximum tolerated dose. At each capsaicin concentration four single-breath inhalations were administered from a dosimeter $30 \mathrm{~s}$ apart. After each inhalation, the number of coughs in the first $15 \mathrm{~s}$ was counted. Using Generalised Estimating Equations (GEE), we explored the effect of group and gender on average cough response to increasing concentrations of capsaicin.

Results We studied 20 treatment resistant CC, 18 stable mild/ moderate $\mathrm{A}$ and $20 \mathrm{HC}$ subjects, matched for age and gender; (M:F $10: 10,9: 9,10: 10$, respectively), age $[$ mean $( \pm S D) 57.1$ years $( \pm 15.7)$,

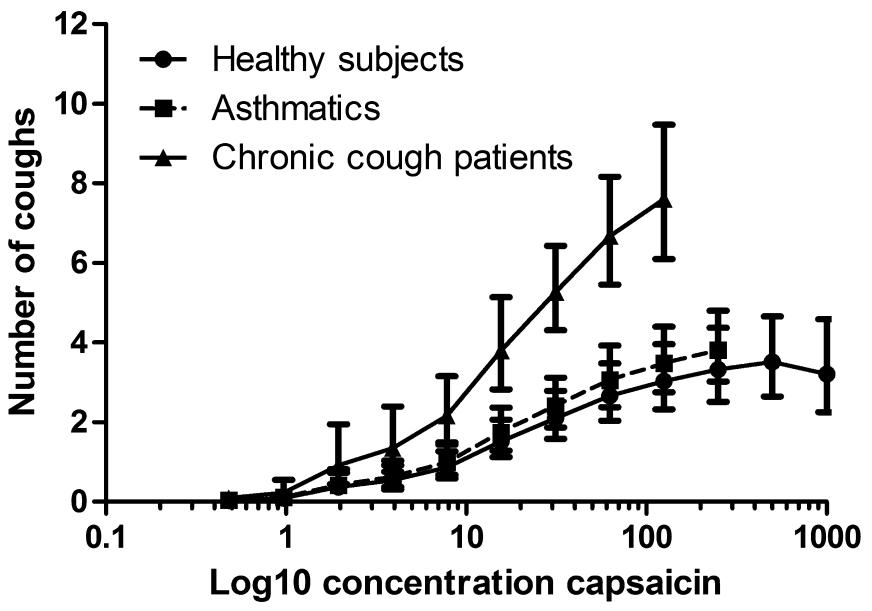

Abstract S115 Figure 1 Average cough frequency with increasing doubling doses of capsaicin (log 10 scale) according to group. Adjusted means and $95 \% \mathrm{Cl}$ from GEE model shown.
51.7 years $( \pm 13.5), 58.8$ years $( \pm 13.5) ; \mathrm{p}=0.17]$, and lung function [3.02L $( \pm 0.98), 2.96 \mathrm{~L}( \pm 1.10), 3.20 \mathrm{~L}( \pm 0.99) ; \mathrm{p}=0.72$ ]. CC (vs HC $\mathrm{p}<0.001$ ) and females (vs males, $\mathrm{p}<0.001$ ) cough significantly more after capsaicin (see Abstract S115 Figure 1). There was no significant difference between $\mathrm{A}$ and $\mathrm{HC}(\mathrm{p}=0.407)$.

Conclusions Magnitude of cough responses to inhaled capsaicin, beyond the standard endpoints:

- clearly discriminates chronic cough from healthy controls,

- demonstrates gender differences,

- may be valuable for investigation of physiological mechanisms and anti-tussive agents.

In addition to a reduced cough threshold, these findings are consistent with a failure of inhibitory mechanisms to prevent coughing to repetitive and potent stimuli in chronic cough.

\section{S116 URGE-TO-COUGH INTENSITY IN CHRONIC COUGH PATIENTS COMPARED TO ASTHMATICS AND HEALTHY CONTROLS}

doi:10.1136/thx.2010.150946.17

${ }^{1} \mathrm{E}$ C Young, ${ }^{1} \mathrm{H}$ Sumner, ${ }^{2} \mathrm{~A}$ Kelsall, ${ }^{1} \mathrm{~A}$ A Woodcock, ${ }^{1} \mathrm{~J}$ A Smith. ${ }^{1}$ University of Manchester, Manchester, UK; ${ }^{2}$ University Hospitals South Manchester NHS Foundation Trust, Manchester, UK

Background Healthy subjects inhaling increasing doses of capsaicin perceive an urge-to-cough (UTC) prior to initiating coughing. We compared subjective ratings of UTC intensity following inhalation of capsaicin in patients with chronic cough (CC), asthma (A) and healthy controls (HC).

Methods A double-blind, randomised block design, capsaicin cough challenge was performed using single-breath inhalations, separated by $1 \mathrm{~min}$, through a dosimeter (inspiratory flow rate limited). Seven individually pre-determined doses $(0.48-1000 \mu \mathrm{Mol}$; maximum dose inducing an average of at least five coughs) and one placebo dose were administered four times each, in four individually randomised blocks (Davenport et al), to reduce the bias associated with subjective UTC ratings. After each inhalation, UTC was rated on a modified Borg scale (0-10) and number of coughs in the first $15 \mathrm{~s}$ was recorded. Using Generalised Estimating Equations (GEE), we explored the effect of group and gender on average UTC intensity and average cough responses to increasing concentrations of capsaicin.

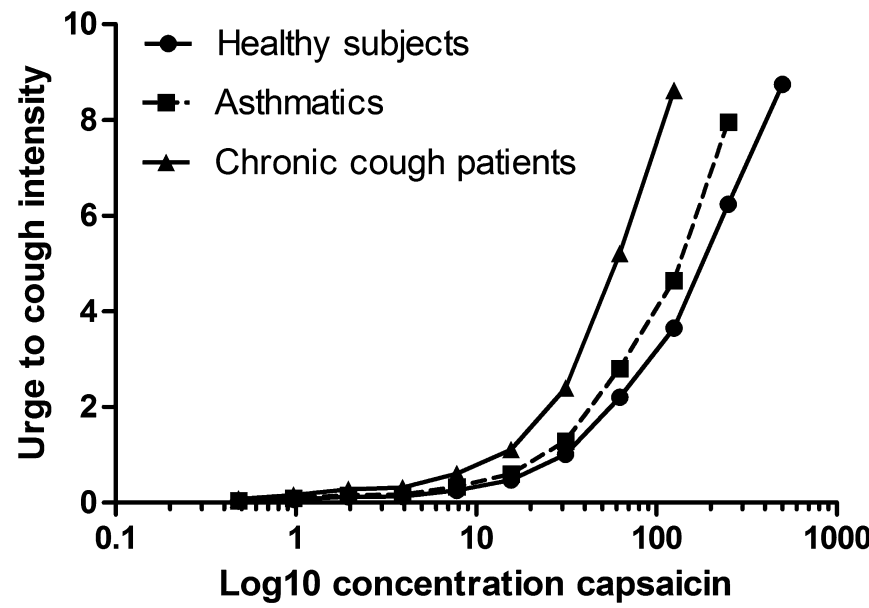

Abstract S116 Figure 1 Average urge to cough frequency with increasing doubling doses of capsaicin (log 10 scale) according to group. Adjusted means extracted from GEE model shown. 